\title{
The Enhanced Measurements of Laser Micro-Jet Processing
}

\author{
Xizhao Lu, Haihe Xie, Chun Lin, Yuanqing Huang \\ Department of Mechanical \& Electrical Engineering Xiamen University, Xiamen, China \\ E-mail:yqhuang@xmu.edu.cn \\ Received November 15, 2010; revised January 11, 2011; accepted January 13, 2011
}

\begin{abstract}
In the paper, the enhanced measurements of the laser micro-jet processing are discussed. In fact, within pure water breakdown threshold of laser, the less focal which focused with the appropriate focusing lens and the small nozzle of water chamber enhance the laser power density , at the same time, the laser beam transport in the wave guided water with the proper total reflection angle. The laser power which depended on the properties of the diameter, the coupling water chamber and the coupling efficiency of the micro-jet and laser beam expect of the properties of laser.
\end{abstract}

Keywords: Focusing Lens, Focal Spot Size, Nozzle of Water Chamber, Laser Micro-Jet, Flat-Topped, Breakdown Threshold

\section{Introduction of Laser Micro-Jet Processing}

Talking about the methods of precise processing, the laser micro-jet system became a hot topic in recent years. The laser micro-jet is utilized in the precise processing, such as semiconductors, MEMS, hard metal, surgical operation and medical stents etc. [1]. More and more practical applications would be developed. This technology needed to overcome the difficulty consisted in two elements-the micro-jet processing and the laser processing. While the diameter of the nozzle was large enough to the laser wavelength, the micro-jet was comparable to a multimode fiber.

In this paper, a new focusing lens with the appropriate bulk of nozzle of water chamber would reform the focal spot into a less one. As a result, the higher power density can be obtained. Micro-jet with stability fluent like pure water is adopted in order to achieve a processing sharp cutter.

It shall be a more focal laser beam in water micro-jet processing. The experiment of laser micro-jet is carried out to make reliable proof. The design of optical path integrated the focusing lens would reduce the focal spot size within the breakdown threshold. The quality of working laser beam was improved with compressing the width of flat-topped of laser beam. During the laser processing, laser micro-jet could be considered as laser wave guided, which dues to the total internal reflection at the water-air interface [2].

Above all, the character of laser beam and the stability water-jet pressure are the key technologies of micro-jet. The liquid fiber with variable length was available with some certain stabile pressure applied into water chamber, while the length of water column depended on the pressure and the design of water chamber and the nozzle. The laser beam is fully reflected at the water-jet surface, similar to transmit the laser within a fiber.

The structure of focusing lens is showed in the Figure 1. The focusing lens will get a small diameter beam spot.

The principle of water-jet guided laser is shown in Figure 2.

The good laser is focused and collimated into a nozzle while passing through a low pressure water chamber and then laser beam was projected to the surface of working

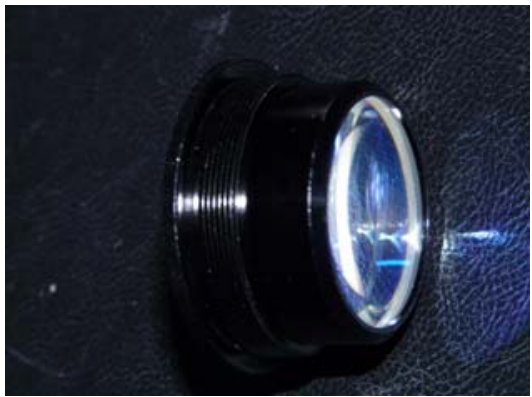

Figure 1. The focusing lens applied in the system. 


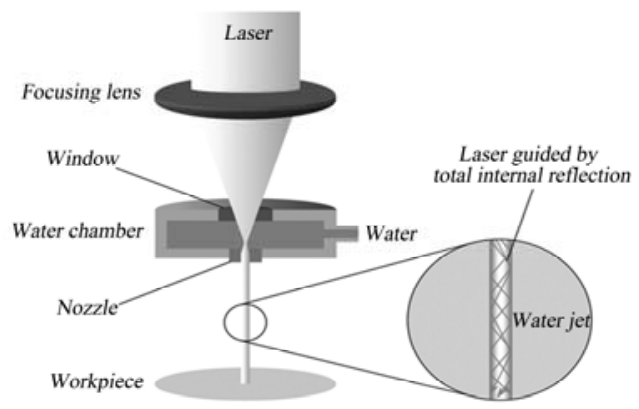

Figure 2. The principle of laser micro-jet [4].

piece. The total reflection angle is $41.25^{\circ}$ [5]. If the design is good, we can couple the beamThe low-pressure water beam was emitted from the nozzle guided the laser beam by means of total internal reflection in the water column, in a manner similar to that of glass fibers. When system are performing the cutting function, the material melts absorbing the laser energy, at the same time, the water under low pressure removed the molten from the cutting work piece,

\section{The Distribution of Micro-Jet Laser is Reformed by the Integrated Nozzle and Focusing Lens}

From the Figure 2 the working laser beam output the single mode or the multi mode is shaped by inverted Galileo telescope. After that, the beam is collimated with a appropriate convex lens. Pure water with adjustable pressure is needed in the laser micro-jet system. The pure water which owns a higher breakdown threshold is necessary [5], too. In fact, the single mode laser was not very often utilized in the processing. In the experiments, the LCS2 000 Laser Optical Analyzer and a reformative water chamber which owned a $300 \mu \mathrm{m}$ nozzle in Figure 3 which own N/A $=50 / 3$ are utilized. The wavelength of working Nd:YAG laser is $1064 \mathrm{~nm}$.

The laser beam was described by well-known relationship:

$$
I(r)=I_{\max } \mathrm{e}^{-2 \frac{r^{2}}{\omega_{o}^{2}}}=\frac{2}{\pi \omega_{o}^{2}} \mathrm{e}^{-2 \frac{r^{2}}{\omega_{o}^{2}}}
$$

where $\omega_{0}$-waist radius of laser beam,

$\mathrm{r}$ - current radius,

I(r) - laser intensity function.

That total energy of laser beam is 1 .

From the Formula (1), I $(r)$ was increasing when $\omega_{0}$ is increased or the value of $\mathrm{r}$ is decreased.

\section{The Experiments of a Focusing Lens and Enhanced the Laser Micro-Jet Beam.}

The energy distribution of laser beam was shown like
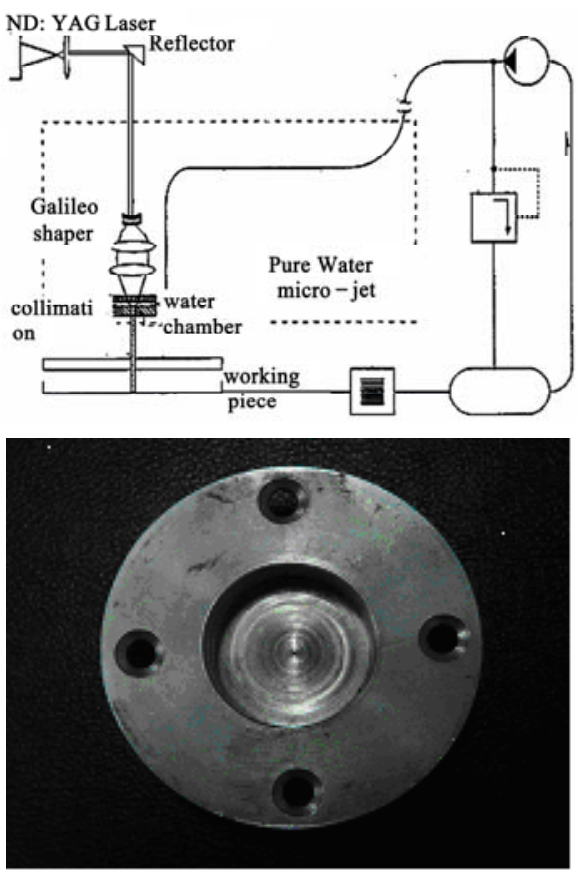

Figure 3. The structure of the laser micro-jet system with focusing lens and nozzle of water chamber.

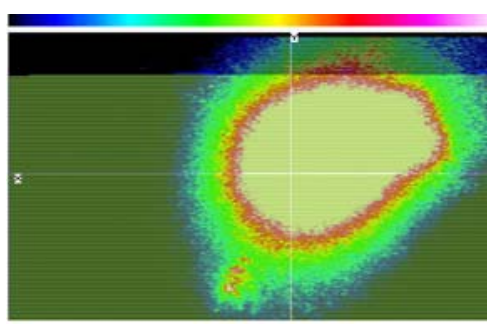

(a)

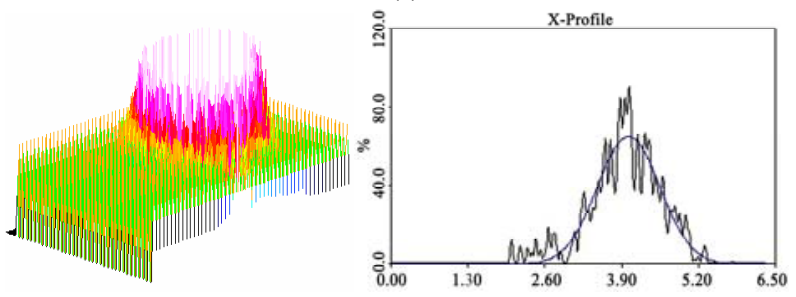

(b)

Figure 4. a) The Nd:YAG laser coupled into the chamber and nozzle without water; b) The $3 \mathrm{D}$ distribution model (left) and x-profile (right) of Nd:YAG laser.

Figure 4(a) and 4(b), when the Nd:YAG laser beam is coupled into the nozzle through chamber without water under some certain pressure.

When the laser beam was coupled in the water chamber, the quality of laser was ameliorated.

To concentrate the collimation laser before transmission into the water chamber, the width of waist radius was about $16 \mu \mathrm{m}$. The flat width of flat-topped laser micro-jet is about $26 \mu \mathrm{m}$ (under very low pressure, such as 


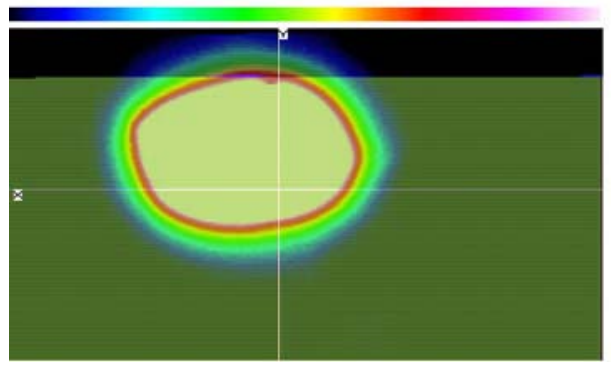

(a)

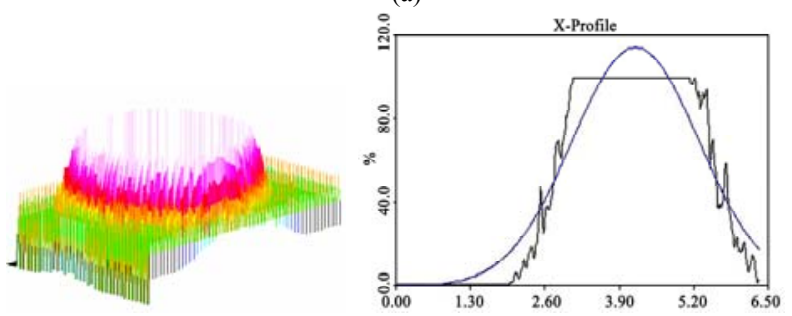

(b)

Figure 5. (a) The Nd:YAG laser coupled in the micro-jet; (b) The laser 3D distribution model (left) and X-profile (right) in water.

\section{$0.1 \mathrm{Mpa})$}

In the Figure 6, some groups of lenses included the Galileo shaper integrated into the water micro-jet system are composed of some convex lenses and a concave lens. Part of them worked like a Galileo telescope. The concave lens would expand the laser. When those were in appropriate positions, it would work as shaper which was the good beam shaper. The system would average the intensity of laser output before concentrated into water chamber and nozzle, that is to say, it was another enhancement the stability of laser beam transmission in micro-jet and minimal the heated affect core zone on the surface of work piece in water column.

The Figure 6 showed the experiment equipment to test the enhancement of quality of working laser micro-jet. In system, the Galileo telescope and the focusing lens enhances the quality of laser worked as Figure 6.

After that, the performance of laser beam in the Figure 5(a) and Figure 5(b) were obtained through the Galileo shaper and then the nozzle in water chamber. Being the diameter of nozzle was $0.03 \mathrm{~mm}$ that power intensity was less than $0.1 \mathrm{~mm}$ which was difficulty to machine but owned some good performance in the experiment. Above all, the laser spot would augment with the increasing diameter of water column. That means the same energy will increase the power intensity, as a result, the power intensity would be lower than breakdown threshold, which should banish the producing bubble in time.

The width of waist laser radius is about $24 \mu \mathrm{m}$, the edge is sharper than the laser micro-jet (about $10 \mu \mathrm{m}$ ).

The consequence of the experiment of laser micro-jet

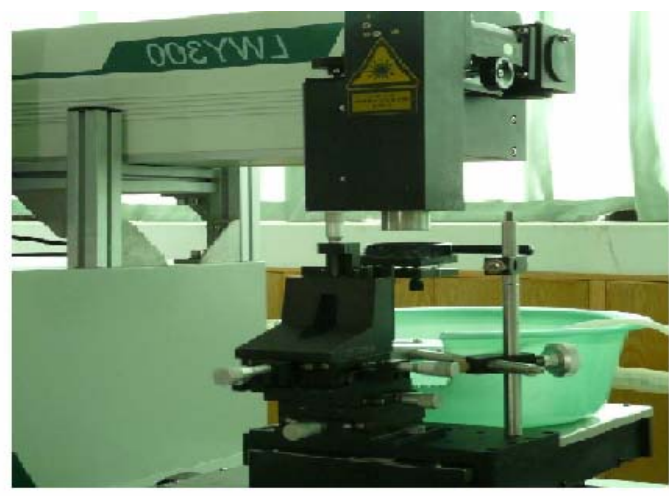

Figure 6. The theoretical experiment system.

which equipped the laser shaper showed the diameters of waster laser radius was less than the one without shaper. We can the consequence of working laser which was made effect on the working piece depended on the factors which not only including peak of laser power but also the power intensity [6]. In some sense, the power intensity was more important than others to improve the quality of laser processing. The spots would be bigger than the one in traditional micro-jet without the shaper. From the experiment and the Formula (1), it is no denying that the diameter of waster laser was reduced and the intensity of laser power would increase.

$$
W=P^{*} T
$$

$W$ - Pulse energy of working laser.

$P$ - Power of laser pulse.

$T$ - The width of laser pulse.

Through the expression (2), the conclusion of the theoretical experiments was draw. Selecting the appropriate energy and the power needed to be lower than the breakdown threshold played an important role as well [5].

\section{The Conclusion of Experiment}

The laser micro-jet is a very fine and precise technique. That stability water-jet is an important role to make a successful processing. The appropriate pressure will enhance the stability of processing. It can cool the laser working condition which the laser would cut the work piece more area with high efficiency.

It was obvious that some certain pressure would give some aspects advantages of laser processing. With enough power intensity of processing form fine kerfs, that is to say, the methods of shaper before the laser coupled into nozzle of water chamber was still efficient to processing in laser micro-jet.

The laser micro-jet with the focusing lens is efficient in making a smooth cutting to enhance the processing efficiency than before. 


\section{References}

[1] J. Battaglia and T. A. Mai, "Delphine Perrottetand Bernold Richerzhagen, Water Jet as a Multimode Waveguide Theoretical and Experimental Investigation of Modal Noise and Beam Propagation in Material Processing with Laser Micro-Jet," 25th International Congress on Applications of Laser and Electro-Optics, 2006.

[2] B. Richerzhagen, "Method and Apparatus for Machining Material with a Liquid guided Laser Beam," United States Patent, 1999, pp. 1-8.

[3] N. Dushkina, F. R. Wagner, C. Boillat, J.-M. Buchilly and B. Richerzhagen, "Water Jet Guided Laser vs. Saw Dicing, Photon Processing in Microelectronics and Photonics II," 2003, pp. 75-85.

[4] B. Richerzhagen, R. Housh and F. Wagner, "Water Jet Guided Laser Cutting: A Powerful Hybrid Technology for Fine Cutting and Grooving," G. Henkelman, G. Johannesson and H. Jónsson, "Theoretical Methods in Condencsed Phase Chemistry," In: S. D. Schwartz, Ed., Progress in Theoretical Chemistry and Physics, chapter, Vol. 5, Kluwer Academic Publishers, Boston, 2000.

[5] C. Xiao, "Studies on Mechanisms of the Interaction between High Power Laser and Matter in Water," Ph.D. Dissertation, Nanjing University of Science \& Technology, China, 2004.

[6] Z. H. Wang, J. Zhan, J. A. Zhen, P. Wang, Z. Y. Wei and J. Zhang, "Ultrashort Laser Wavefront Correction, SCIENCE IN CHINA Ser. G Physics," Mechanics \& Astronomy, Vol. 34, No. 6, 2004, pp. 620-627.

[7] L. Li, "Study on Water-Jet Guided Laser Micromachining Technology," Ph.D. Dissertation, Harbin Institute of Technology, Harbin, 2008. 